\title{
BEHIND THE SCENES OF MUNCHAUSEN SYNDROME BY PROXY: 'You know I love you, right?
}

WORLD

PSYCHIATRIC

ASSOCIATION
Tânia B Cavaco ${ }^{1}$, Raquel Campos ${ }^{1}$

${ }^{1}$ Child and Adolescent Psychiatry Resident, Garcia de Orta Hospital (Almada, Portugal) Institutional e-mai: tania.cavaco@hgo.min-saude.pt $19^{\text {TH }}$ WPA WORLD

CONGRESS OF PSYCHIATRY LISBON, 21-24 AUGUST, 2019

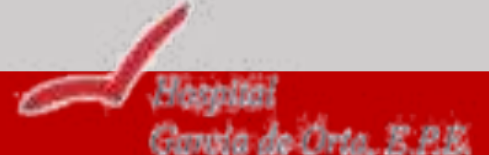

\section{INTRODUCTION}

- Munchausen syndrome by proxy (MSBP), often termed Medical Child Abuse or Factitious Disorder Imposed on Another, is a form of child maltreatment in which a child receives unnecessary and potentially harmful medical care at the instigation of a caregiver who intentionally induces or simulates symptoms/ signs in a child, associated with fulfilment of the abuser's own psychological need for gratification;

- MSBP may lead to significant neurodevelopmental harm to the child, global health problems or death;

- It is fundamental to identify these cases of abuse by efficiently recognising the perpetrators' profile and the most common red flags in MSBP, allowing for prompt protective measures.

METHODS

Non-systematic review of original and review articles, indexed on PubMed, selected by relevance and language (English and Portuguese), using the keywords: Munchausen syndrome by proxy, medical child abuse, factitious disorder imposed on another. Articles referenced on the bibliography were also included.

\section{RESULTS}

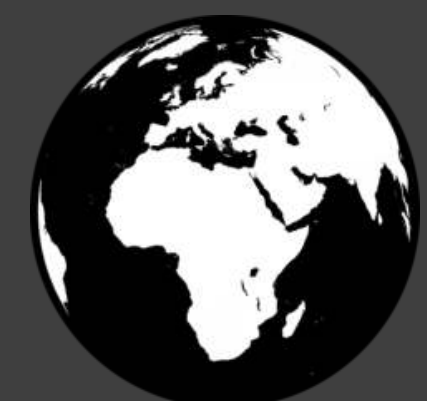

The reported annual incidence of MSBP is $0.4 / 100^{\prime} 000$, for children under 16 y.o.,

and $2 / 100$ '000, for children under 1 y.o.

MSBP might result in high paediatric morbidity and mortality (6-9\%).

The perpetrators are mostly:

* Female (92-100\%)

* Young (25-31 y.o.)

* Married (43-79\%)

* Mother of the child (76.5-100\%)

* Workers in healthcare ( $46 \%)$
Perpetrators often have multiple METHODS OF ABUSE: Fabrication by words, by hands (simulation $21.7 \%$, induction $57.4 \%$ ). Most times fabrication continues during hospitalization (54.4\%).
Many have a PSYCHIATRIC HISTORY: Munchausen Syndrome, $\mathrm{hx}$ of child maltreatment, personality/mood disorders, depression, substance or alcohol abuse, physical or sexual abuse, obstetric complications.

Several features of the medical evaluation may elicit SUSPICION OF MSBP, such as :

$\checkmark$ Discordant history with objective findings, unexplained or unexpected symptoms/signs, child's atypical response to treatments or caregiver insistence for more medical interventions/exams.

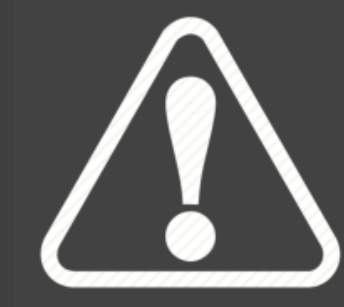

Many times, direct confrontation of the abuser has led to the suicide of the perpetrator.

\section{CONCLUSIONS}

* Although MSBP appears to be less common than most forms of child maltreatment, it seems to be more lethal;

* Early recognition and management of this syndrome is crucial, since physicians and the healthcare system may also unintentionally serve as harmful agents to the child. Consultation with a child protection team or other providers with advanced training and expertise in child maltreatment should be considered upon suspicion;

* The aim is to repair the physical and psychological damage in the least restrictive manner that still ensures the child's safety;

* Recovery may involve reversal of medical procedures and intensive psychotherapy, although some children will suffer from long-term or permanent trauma or injury. 